\title{
Tecnología y enseñanza: retos y nuevas tecnologías y metodologías*
}

Technology and teaching: challenges and new technologies and methodologies

Citar como: Cabero-Almenara, J. (2020). Tecnología y enseñanza: retos y nuevas tecnologías y metodologías. CITAS, 6(1). https://doi.org/10.15332/24224529.6356

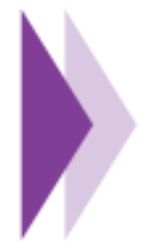

Julio Cabero-Almenara ${ }^{1}$

1 Organización Escolar de la Universidad de Sevilla

Correoelectrónico: cabero@us.es 


\section{Resumen}

En este artículo se exponen los principales acontecimientos que dieron origen a la Sociedad de la Información (SI) y el Conocimiento (SC) y se reflexiona sobre cómo estos han transformado diferentes aspectos sociales, económicos y productivos; principalmente, se resalta la influencia de la tecnología en los procesos de enseñanza y aprendizaje en contextos educativos. En la primera parte, se realiza un recorrido por la evolución de la industria, iniciando por la sociedad agraria hasta la Industria 4.0 y la transformación digital. En la segunda parte, se presentan las Tecnologías de la Información y Comunicación (TIC) como los nuevos escenarios para el aprendizaje y la formación, con un análisis de las tecnologías que se están utilizando y que se encuentran en los informes Horizon. Finalmente, se reiteran los beneficios de la implementación de las tecnologías como mediadoras en los entornos didácticos y pedagógicos.

Palabras clave: conocimiento, enseñanza, información, metodologías, sociedad, tecnologías.

\section{Nuevos escenarios para la formación: la sociedad del conocimiento}

Las definiciones realizadas por la Sociedad de la Información (SI) y el Conocimiento (SC) han sido diversas, pero todas estas giran alrededor de indicar que es un nuevo estadio de desarrollo social que supera a los anteriores - agrícola e industrial, y posindustrialy que viene determinado por la capacidad que tienen las personas e instituciones que en este se desenvuelven por obtener, compartir y procesar cualquier información, desde cualquier lugar y en una diversidad de formatos y sistemas simbólicos.

Dicha sociedad se caracteriza por una serie de hechos distintivos como son: la globalización económica y cultural, la velocidad con que se genera y transforma la información, la necesidad de movernos en un aprendizaje constante y la ruptura de la concepción tradicional que tenemos de los conceptos de espacio y tiempo. Es decir, es una sociedad que requiere el aprendizaje constante, una sociedad de redes no solo de individuos, que se apoya en modelos económicos centrados en la información y la amplitud de TIC que tenemos a nuestra disposición (Cabero, 2016).

Tal situación nos introduce en un contexto ambiguo, complejo, dinámico y desconocido, claramente diferente al de la sociedad posindustrial, donde todo tendía a estar regulado y estandarizado. Como dijo el filósofo Bauman (2007), estamos en un mundo de la modernidad líquida, donde todo es rápido y perenne. Brynjolfsson y McAfee (2014) han llamado esta época la segunda edad de las máquinas, que presenta una diferencia fundamental con respecto a la primera: esta se basó en las máquinas de vapor para superar los límites físicos de los humanos y los animales, mientras que la actual se apoya en las tecnologías digitales para superar los límites de las capacidades intelectuales humanas.

De manera específica, Valencia (2018) indica que hoy

[...] en día vivimos en un mundo VUCA (Volatility, Uncertainty, Complexity, Ambiguity, por sus siglas en inglés, o sea, Volátil, Incierto, Complejo y Ambiguo), con dinámicas de cambio constante y difícil de predecir, con multiplicidad de fuerzas que se interrelacionan en todos los ámbitos del conocimiento y la vida, con una interdependencia de variables difíciles de descifrar, con

\section{Abstract}

This article presents the main events that gave rise to the Information Society (IS) and Knowledge Society (KS) and reflects on how they have transformed different social, economic and productive aspects; mainly, it highlights the influence of technology on teaching and learning processes in educational contexts. In the first part, a journey is made through the evolution of industry, starting from the agrarian society to Industry 4.0 and digital transformation. In the second part, Information and Communication Technologies (ICTs) are presented as the new scenarios for learning and training, with an analysis of the technologies being used and found in the Horizon reports. Finally, the benefits of the implementation of technologies as mediators in didactic and pedagogical environments are reiterated.

Keywords: knowledge, teaching, information, methodologies, society, technologies.

oportunidades y desafíos permanentes que implican el manejo constante de alternativas que saltan día a día en nuestra realidad.

Lo verdaderamente importante de esta situación en la que nos movemos es que nos encontramos en una posición de cambios constantes respecto a los estadios anteriores; estos se producen en diferentes sectores, que van desde los sociales a los políticos, y desde los económicos a los laborales. Como indica West (2016), nos encontramos en una época de megacambio, lo que repercute en que los patrones sociales, económicos y políticos han dejado de ser fijos; esto produce inseguridad, así como miedo a los otros y al futuro. Esta situación marcará completamente la situación profesional de los docentes, ya que antes todo estaba perfectamente regulado y controlado, se sabía qué, quién y cómo debía aprender o la durabilidad de la información aprendida.

Ello exigirá que asumamos desde el principio que los cambios que se necesitan en la SI no son solo tecnológicos, sino también organizativos y culturales. De todas formas, debemos ser conscientes de que los cambios tecnológicos

[...] aceleran o multiplican los efectos de la globalización. La mejora de las telecomunicaciones, la conectividad, internet, etc., alimentan la globalización económica, política y cultural. Por otra parte, los mercados globalizados son el marco natural de la revolución tecnológica, en el que puede desarrollar todas sus potencialidades (González, 2017, p. $15)$.

Estos cambios repercutirán en el mercado del trabajo. De hecho, García (2017) señala un informe de 2016 de CaixaBank denominado Las nuevas tecnologías y el trabajo, donde se expone, con claridad, que el $43 \%$ de los puestos laborales actuales desaparecerán en los próximos años, debido a la robotización y utilización de inteligencia artificial. Dichas transformaciones tecnológicas también afectan "nuestro sentido del yo, cómo nos relacionamos con los demás y cómo damos forma e interactuamos con nuestro mundo" (Floridi, 2017, p. 8).

Ante esta situación, y como señala la Unesco (2015, p. 3), si "el mundo está cambiando: la educación debe cambiar también. Las sociedades de todo el planeta experimentan profundas transformaciones y ello exige nuevas formas de educación que 
fomenten las competencias que las sociedades y las economías necesitan hoy día y mañana".

Tal necesidad de cambio se justifica por diversos motivos. Por una parte, porque la durabilidad de los contenidos y el volumen de información no es la misma que anteriormente, cuando era suficiente para una persona lo que aprendía o retenía en su período formal de instrucción, con mínimas adaptaciones, para desenvolverse durante gran parte de su vida profesional y personal. Y por otra, porque ante esta situación, las instituciones escolares estaban pensadas para dar respuestas correctas y duraderas, y en la actualidad ello no es posible, puesto que ya no existe lo correcto, seguro ni perpetuo.

Antes había una edad para aprender y analizar contenidos predefinidos en un tiempo claramente regulado. Todos los alumnos estaban ubicados en el mismo sitio y todas las personas tenían que aprender las mismas cosas. En la sociedad del conocimiento esto ya no es posible, pues el aprendizaje se convierte en una necesidad constante para la persona, donde gracias a la tecnología se permite la individualización y la potenciación de una enseñanza personalizada. Ello repercute en la creación de una nueva ecología del aprendizaje, ya que empezamos a percibir la formación en un sentido amplio y no limitado a un período escolar concreto.

Cada vez resulta más complejo imaginarnos cómo será el futuro, y ello es uno de los grandes problemas con que se enfrentan los docentes en la actualidad, pues tal dificultad repercute en determinar cómo formaremos a los estudiantes. Por otro lado, si imaginamos el futuro lo hacemos mirando por un retrovisor, o como dirá Jarvis (2015, p. 12): "Si hoy definimos el futuro, lo hacemos en términos del pasado". De ahí que la profesión docente sea una de las más críticas para el desarrollo de la sociedad del conocimiento.

Por otra parte y como apuntan Loveless y Williamson (2017) la tecnología y la educación conforman un sistema sociotécnico. Este sistema reconoce que la tecnología y la sociedad son mutuamente constitutivas; la tecnología influye en las relaciones sociales, mientras que las relaciones sociales influyen en el desarrollo y la adopción de tecnologías.

Pero, además, aunque ahora solo lo apuntaremos y lo abordaremos al final de nuestro trabajo, los estudiantes que entran en nuestras aulas son distintos a las épocas pasadas: tienen motivaciones diferentes, interaccionan de manera distinta y poseen habilidades cognitivas desemejantes a las que teníamos nosotros.

Como señala Pérez Gómez (2012, p. 71):
La sociedad de la información y del conocimiento dirige a la educación demandas distintas de las tradicionales, claramente relacionadas con el desarrollo en todos los ciudadanos de la capacidad de aprender a lo largo de toda la vida. Dicho de otro modo, el problema no es ya la cantidad de información que los niños y jóvenes reciben, sino la calidad de la misma: la capacidad para entenderla, procesarla, seleccionarla organizarla y transformarla en conocimiento; así como la capacidad de aplicarla a las diferentes situaciones y contextos en virtud de los valores e intenciones de los propios proyectos personales o sociales.

Como acertadamente destaca Valencia (2017):

Necesitamos educar a la gente para los trabajos que aún no han sido creados, para usar tecnologías que aún no han sido inventadas, para resolver problemas económicos y sociales que aún no surgen. Este es el reto fundamental para la educación. En resumen, el mundo no pagará más por lo que sabes, pues ya Google lo sabe todo. Los egresados cobrarán por lo que son capaces de hacer con lo que saben.

Además de esta situación de complejidad e incertidumbre que hemos apuntado, nos encontramos con diferentes hechos que influyen directamente en la necesidad de transformación del sistema educativo, como son: la fuerte presencia de las TIC en la sociedad, la amplitud de información que tenemos a nuestra disposición y la extensión de escenarios de aprendizaje. En el análisis de estos elementos nos centraremos a continuación.

Las tecnologías de la información y la
comunicación (TIC) en la configuración de
nuevos escenarios para el aprendizaje y la formación

Por lo que se refiere a la primera, y centrándonos en la institución escolar, tenemos que señalar que nunca han existido tantas TIC como en los momentos actuales; es más, en el futuro cercano estas se verán fuertemente ampliadas, como ponen de manifiesto los diferentes informes Horizon o los reportes EduTrends del Tecnológico de Monterrey (2015). En la tabla 1 están las propuestas realizadas por diferentes Informes Horizonte, específicamente los elaborados para el contexto iberoamericano, las tecnologías que allí se apuntaban que tendrían un horizonte de penetración en las instituciones educativas, a 1 año o menos, a de 2 a 3 años y 3 a 6 años. 
Tabla 1. Penetración de tecnologías en la institución educativa según diferentes Informes Horizon

\begin{tabular}{|c|c|c|c|}
\hline Informes Horizon & A un año o menos & De 2 a 3 años & De 3 a 6 años \\
\hline García et ál. (2010). & $\begin{array}{l}\text { Entornos } \\
\text { colaborativos } \\
\text { Medios sociales }\end{array}$ & $\begin{array}{l}\text { Contenidos abiertos } \\
\text { Móviles }\end{array}$ & $\begin{array}{l}\text { Realidad aumentada } \\
\text { Web semántica }\end{array}$ \\
\hline Durall et ál. (2012). & $\begin{array}{l}\text { Contenidos abiertos } \\
\text { Aplicaciones móviles } \\
\text { Computación en la } \\
\text { nube } \\
\text { Entornos } \\
\text { colaborativos }\end{array}$ & $\begin{array}{l}\text { Tabletas } \\
\text { Aprendizaje basado } \\
\text { en juegos } \\
\text { PLE } \\
\text { Geolocalización }\end{array}$ & $\begin{array}{l}\text { Analíticas de } \\
\text { aprendizaje } \\
\text { Realidad aumentada } \\
\text { Web semántica } \\
\text { MOOC }\end{array}$ \\
\hline Johnson et ál. (2013) & $\begin{array}{l}\text { Contenidos abiertos } \\
\text { Aprendizaje en línea } \\
\text { Entornos } \\
\text { colaborativos } \\
\text { Medios sociales }\end{array}$ & $\begin{array}{l}\text { Realidad aumentada } \\
\text { Aprendizaje móvil } \\
\text { Aprendizaje } \\
\text { personalizado } \\
\text { Analíticas de } \\
\text { aprendizaje }\end{array}$ & $\begin{array}{l}\text { Aprendizaje } \\
\text { aumentado } \\
\text { Impresión en } 3 \mathrm{D} \\
\text { Internet de las cosas } \\
\text { Laboratorios virtuales } \\
\text { y remotos }\end{array}$ \\
\hline
\end{tabular}

Fuente: elaboración propia.

Independientemente de este volumen de tecnologías, lo importante es no perder de vista que cada vez nos encontramos con más tecnologías emergentes que, como señalan Sosa, Salinas y de Benito $(2017,120)$, se "refieren a recursos, artefactos, herramientas, conceptos e innovaciones, asociados a lo digital, que tienen un potencial disruptivo de transformar o generar cambios en los procesos donde se utilizan sin importar si estas son nuevas o viejas tecnologías". Desde esta perspectiva, no cabe la menor duda de que tecnologías como la realidad aumentada, las analíticas de aprendizaje y el internet de las cosas revolucionarán y transformarán toda la realidad educativa. Como señala Mas (2017), la red se convertirá en un entorno de aprendizaje abierto, ya que "la evolución de internet y de los usos de la tecnología digital de las personas conduce actualmente a la proliferación de formas de aprendizaje abiertas, donde las fronteras entre formal e informal tienden a desdibujarse" (Mas, 2017, pp. 18-19).

Respecto a su aumento, tampoco se puede obviar que es cierto que la innovación tecnológica no está uniformemente distribuida, que no ocurre en todos los lugares de la misma forma, que existe una brecha digital entre países, zonas y centros, ni tampoco que los periodos de acercamiento tecnológico son cada vez más cortos que en otros momentos históricos, entre otros motivos, porque desde las propias administraciones de políticas educativas se percibe la urgencia de incorporación a la formación. Valga como ejemplo cómo la Organizacio\#n de Estados Iberoamericanos (OEI) en sus Metas para el 2021 en Latinoamérica, señala que las TIC

[...] han ido ocupando un lugar de importancia como recurso educativo de indispensable incorporación en el sistema educativo. Las TIC, y especialmente el acceso al computador y la conexión a internet, son recursos innovadores que han demostrado ser necesarios, además, como herramientas para desenvolverse en un mundo posmoderno y globalizado. Actualmente, la inclusión social se vincula, cada vez más, con el acceso al conocimiento, por la participación en redes y por el uso de las TIC. (OEI, 2010, p. 71).

Por lo tanto, podemos decir que llegaremos todos; otra cosa diferente es si llegaremos todos a tiempo y la brecha digital, en lugar de reducirse, se siga ampliando por su relación con la desigualdad social.

Asociado con la expansión de las TIC, nos encontramos con el aumento de la información, ya que nunca en la historia de la humanidad se había generado tanta información, a tal velocidad, como en los momentos actuales. Tünnerman (2009) señala que si el conocimiento se demoró 1750 años en duplicarse por primera vez, contando desde el inicio de la era cristiana, luego se duplicó cada 150 años y ahora lo hace cada cinco años; se estima que para el 2020 se duplicará cada 73 días. Otro ejemplo de tal velocidad nos lo indican Cross y colaboradores (2010), al señalar que en 1985 el $75 \%$ de los conocimientos necesarios para trabajar los teníamos en nuestra cabeza y hoy ni siguiera llegamos al $10 \%$. Por ello necesitamos apoyarnos en dispositivos tecnológicos (USB, smartphones) para guardar la información y utilizarla cuando la necesitemos; es lo que empieza a denominarse como inteligencia ambiental.

Frente a los momentos anteriores donde la información era rígida, individual y duradera, en la actualidad viene marcada por diferentes características: abierta, distribuida, escalable, volátil, social, en redes, global, adaptativa y autoorganizada. Lo cual también nos lleva a que la formación de la ciudadanía en esta era digital se debe abordar desde miradas diferentes. Como señala García (2017, p. 4), el volumen de la información y su rapidez de generación y destrucción nos llevará a ser nómadas del conocimiento. "En definitiva, en la era digital el conocimiento ha 
cambiado de fondo y de forma. La narración lineal ha dado paso a una estructura en red, multicanal y transmediática, y el objeto sólido, a un fluido dinámico e interconectado" (Mas, 2017, 33).

Más allá de las cifras señaladas anteriormente, tal volumen de información nos introduce en una compleja situación: por una parte, hacer hincapié en crear un sistema educativo donde la función del alumno no sea tanto retener datos, ya que no tiene mucho sentido en un mundo donde la información se transforma constantemente y no tiene la durabilidad de los momentos anteriores, y por otra, como señala Rué (2015):

El valor de estos datos, en el contexto de una sociedad denominada precisamente del conocimiento, es poner de manifiesto que la información es tanta y de acceso tan relativamente fácil, que el valor añadido de la formación ya no consiste solo en saber retenerla y comprenderla, sino que se halla en el modo de buscarla, de tratarla, de elaborarla críticamente y de hacerlo de la forma más relevante y creativa posible en los contextos específicos de resolución de problemas. (pp. 44-45)

De ahí que la formación no deba centrarse tanto en la simple retención de los datos, y con ello no quiero decir que no se potencie la memoria, que es una habilidad cognitiva extraordinariamente importante (Rojas, 2011) y posiblemente no muy bien comprendida en los momentos educativos actuales, sino que se debe potenciar la adquisición de competencias y habilidades. Ello nos debe llevar a repensar el concepto de aprendizaje y las estrategias de enseñanza, pues el alumno como competencias mínimas tendrá que tener las capacidades de aprender, desaprender y reaprender. Aunque esto no es nada fácil, pues todos creemos saber o intuir que hay que cambiar, pero cómo hacer el cambio y en qué dirección no está nada claro y además no hay un acuerdo.

En este contexto, como señala Valencia (2017),

[...] la relación pedagógica entre profesor-alumno requiere de docentes que no solo reproduzcan el contenido de una asignatura, sino que permitan a sus estudiantes extrapolar ese conocimiento hacia el uso y aplicación del mismo en situaciones nuevas. Hay que pensar en un nuevo ecosistema sostenible para la educación superior del siglo XXI, que seguramente será muy diferente al que conocemos hoy.

Por último, nos encontramos en una situación donde las estancias formales de educación están dejando de ser la única forma de adquirir información y construir conocimientos. En los momentos actuales, los contextos informales y no formales aportan tantos escenarios para la formación de la ciudadanía como los tradicionales formales (Salmi, 2012; Unesco, 2015). Conner (2013) nos habla de que nos encontramos en una situación donde los escenarios de formación van desde lo formal a lo informal, y desde lo intencional a lo inesperado; estableciendo combinaciones entre lo formal-inesperado (medios sociales, navegación por internet), lo informal-inesperado (comunidad virtual, juegos), lo formalintencional (clases, e-learning) y lo intencional-informal (lecturas, tutorías).

En definitiva, gracias a las tecnologías el aprendizaje se hace cada vez más ubicuo y descontextualizado de los lugares tradicionales, y más cercano a los momentos temporales y espaciales en los que se necesita. Para ello las TIC pueden ser un elemento clave, más cuando nos desenvolvemos en una sociedad de aprender a aprender o del aprendizaje constante; nos moveremos cada vez más en unos entornos enriquecidos por las tecnologías.
La situación que hemos dibujado nos lleva necesariamente a señalar, por una parte, que los escenarios de formación van a ser muy tecnológicos, o por decirlo con otros términos, van a ser escenarios muy enriquecidos por las TIC; por otra, que el propio proceso de aprendizaje se va a ver transformado. Como señala Prensky (2011): "La problemática no es solo cambiar de tecnología, sino también de la concepción que tengamos del aprendizaje". Por otra parte, como se sugiere en el último informe Horizon para la enseñanza superior (Johnson et ál., 2016), el conocimiento que las personas necesitan para vivir y trabajar en la sociedad actual es crecientemente interdisciplinar, centrado en problemas y basado en procesos. Además, se demandan nuevas habilidades que incluyen la pericia para trabajar en equipo, excelentes destrezas de presentación e intercomunicación, procesos de pensamiento crítico y la competencia digital para gestionar la tecnología y la abundante información con que nos encontramos de forma efectiva. Esto implica crear nuevas modalidades y escenarios formativos, y capacitar a los alumnos en la adquisición de competencias diferentes a las potenciadas en otros momentos.

Tal situación lleva a reclamar la necesidad de crear nuevas modalidades y escenarios formativos y a utilizar nuevas teorías de aprendizaje que permitan justificarlas y comprenderlas. En este aspecto de las teorías del aprendizaje, las constructivistas, conectivistas y cognitivas están aportando algunas concepciones respecto a cómo se produce el aprendizaje en los escenarios tecnológicos, algunas de estas son:

- Es un proceso activo y no pasivo. Lo que implica pasar del alumno como receptor a desarrollador; es decir, crear escenarios para que pase de consumidor a proconsumidor.

- Los alumnos no solo deben reproducir conocimientos sino también crearlos.

- $\quad$ El aprendizaje es un proceso social y, por lo tanto, tiene bastante importancia el aprendizaje colaborativo.

- Los conocimientos previos que tenga el estudiante son determinantes para la consolidación del aprendizaje, ya que es acumulativo.

- En el aprendizaje están implicados factores cognitivos y metacognitivos, motivacionales, emotivos, sociales y culturales

- La consciencia y capacidad que tenga el estudiante de autorregular su aprendizaje determina el comportamiento de este.

- $\quad$ Es integrado, contextualizado y situado, en función de los problemas, necesidades y ubicación espacial del sujeto.

- No hay una única forma de aprender y, por lo tanto, no existe un único modelo para garantizar su sitio.

- Se deben movilizar diferentes sistemas simbólicos para desarrollar e impulsar los diferentes tipos de inteligencias y así favorecer una educación más personalizada.

- Su evaluación no debe referirse únicamente a los productos, sino a los procesos que lo han generado, no debe limitarse a uno de los actores, los alumnos, sino a todas las variables implicadas en el proceso; lo que implica pasar de una evaluación sumativa a una evaluación auténtica.

De todas formas, debemos ser conscientes de que al lado de las teorías que podríamos considerar como clásicas para explicar el proceso de aprendizaje, están acercándose otras (aprendizaje invisible, aprendizaje ubicuo, aprendizaje rizomático, aprendizaje conectado) (Cabero y Llorente, 2015) que pueden ser de utilidad 
para comprender cómo se produce el aprendizaje en los contextos tecnológicos digitales.

En esta nueva situación de formación, producida por la SI, se requiere adoptar una serie de principios directores como los siguientes:

1. Ubicación del alumno en el centro del proceso de enseñanza y aprendizaje.

2. Un profesor que cambia de rol y que, más allá de seguir transmitiendo conocimiento, pasa de estar centrado en su materia o contenido a estarlo también en el desarrollo humano y personal del alumno, pasa a ser el diseñador de la acción formativa.

3. Un aprendizaje basado en una comunidad educativa de profesores y alumnos, en la relación personal, en la creación de vínculos y en el trabajo cooperativo; es decir, aprender de los otros y con los otros.

4. Un aprendizaje interdisciplinar basado en el planteamiento y la resolución de retos que superan las materias curriculares y están conectados con la vida, la profesión, el entorno y las empresas, y el contexto significativo que rodea al alumno.

5. Una formación con mirada y sentido integrales, dirigida a todas las inteligencias del alumno y orientada a impactar en el conjunto de la persona para ayudarla a conocerse y a construir su proyecto futuro. Además, entre todas las inteligencias contar con la emocional.

Cabría hacer ahora una pregunta: ¿cómo será el aprendizaje del futuro? Lógicamente no tenemos una bola de cristal para evitar el riesgo de equivocarnos, y más en los momentos inciertos y líquidos en los que nos movemos, pero están apareciendo indicios que permiten apuntar algunas ideas al respecto: visual, abierto, social, mezcla de lo real y lo virtual, descontextualizado y ubicuo, personalizado, móvil y enredado.

Todos estaremos de acuerdo en que lo visual va ganando terreno como medio que despierta verdadero interés, como lo demuestra el hecho de que los programas de televisión, específicamente las series, son cada vez más observadas bajo la modalidad de video bajo demanda a través de internet en plataformas específicas como YouTube, más que en el medio tradicional televisivo. También en el caso de la educación a distancia bajo la modalidad de $e$ learning, la investigación ha puesto de manifiesto que la utilización de fragmentos de video la hace más eficaz (Ljubojevic et ál., 2015), o la utilización de estos en estrategias de enseñanza que se están poniendo de moda, como el flipped classroom (Prieto, 2017), sin olvidarnos de las preferencias de las nuevas generaciones por lo visual frente a lo escrito.

El movimiento de los contenidos abiertos (Open Educational Resources) o recursos educativos abiertos crece progresivamente debido a diferentes motivos, que van desde su potenciación desde las estancias oficiales como la Unesco (2013), la promoción institucional del uso de licencias Creative Commons, el movimiento del Open Course Ware (http://ocw.mit.edu) creado por el Instituto Tecnológico de Massachusetts o la significación que están adquiriendo los cursos MOOC.

Por recursos educativos abiertos podemos entender: "materiales digitalizados ofrecidos libre y abiertamente para profesores, alumnos y autodidactas, a fin de que sean usados y reutilizados para enseñar mientras se aprende y se investiga" (RAE, en Organización para la Cooperación y el Desarrollo Económicos [OCDE] y Junta de Extremadura, 2008, p. 14). Esta política ha evolucionado desde los simples repositorios de materiales de enseñanza a los que el profesor podía acceder para utilizar determinados recursos cuando los necesitase, a contenidos más estructurados en formato de cursos completos con tareas a realizar y recomendaciones para la evaluación de los estudiantes. Tal es la importancia de este movimiento, que los Informes Horizon del 2010 (Jonhson et ál., 2010; y García et ál., 2010) lo situaban con una perspectiva de incorporación a educación en un horizonte de un año, y de dos a tres en el caso iberoamericano, planteamiento que es confirmado.

Directamente relacionado con lo anterior nos encontramos con que el aprendizaje será cada vez más ubicuo y móvil, ya que podrá realizarse en cualquier lugar y momento gracias a la diversidad de dispositivos móviles con los que contamos en la actualidad (Castaño y Cabero, 2013; Vázquez-Cano y Sevillano, 2015), lo que facilitará el acceso a la información a la ciudadanía, y contenidos de calidad a personas que se encuentren ubicadas en zonas marginales, sin olvidarnos de la potenciación de una formación justo en el momento que se necesita.

De otro lado, el aprendizaje será al mismo tiempo cada vez más social e individualizado. Social desde la perspectiva de que el aprendizaje en la sociedad del conocimiento requiere la colaboración de diferentes personas para la construcción del conocimiento, en lo que se ha denominado como inteligencia colectiva; es decir, la red se está convirtiendo en un instrumento que mediante los aportes individuales de información y conocimiento propicia la creación de una verdadera inteligencia colectiva y permite crear información más rica y sofisticada que la de las aportaciones individuales. Asociado a este concepto aparece el de aprendizaje colaborativo/cooperativo, mediante el cual el estudiante adquiere el papel de proconsumidor (Rifkin, 2014), producto del cambio producido por la web 2.0, que a diferencia de la web 1.0, el protagonista es el usuario y no los contenidos. En cierta medida podríamos decir que se ha pasado de una sociedad de consumo individual de la información a una sociedad de construcción colaborativa y conjunta del conocimiento. Al mismo tiempo el aprendizaje es individualizado, ya que la educación será cada vez más personalizada, y respetará las características personales, los intereses de los estudiantes y sus inteligencias múltiples, para favorecer la acción educativa (Gardner, 1998).

El carácter social del aprendizaje viene potenciado por la importancia que están adquiriendo las redes sociales, las comunidades virtuales y las comunidades virtuales de aprendizaje. Estas últimas presentan una serie de características: la interacción que se establece entre las personas que conforman la CVA se efectúa a través de tecnologías flexibles en el tiempo, se caracterizan por el intercambio de información en diferentes formatos y por la generación y construcción de conocimientos nuevos; sus participantes comparten un lenguaje y no necesariamente unas creencias; la interacción se realiza utilizando diferentes tipos de herramientas de comunicación sincrónicas, y asincrónicas y textuales como audiovisuales; y se realiza una comunicación multidireccional (Cabero y Llorente, 2010).

No podemos tampoco olvidarnos - $-\mathrm{y}$ ya he efectuado algún comentario al respecto- que el aprendizaje ya no se produce solo en la escuela, cada vez será más ubicuo; es decir, se producirá en cualquier momento y en cualquier lugar. De aquí que la función de la escuela sea más integrar los diferentes aprendizajes que se producen en contextos diferenciados, que, como ocurre en los sistemas educativos tradicionales, potenciar la estandarización y la uniformidad.

Los escenarios futuros de formación serán cada vez producto de la combinación de los escenarios analógicos-reales y los digitalesvirtuales que, en combinación, aportarán nuevas realidades para 
analizar y comprender la información. Ejemplo de lo que hablamos son la realidad aumentada y virtual (Cabero y García, 2016), la primera pretende mezclar la realidad con lo virtual, lo que permite a los usuarios interactuar con los mundos físico y digital; la segunda facilita a los usuarios la inmersión en un mundo alternativo, simulado por ordenador, donde ocurren experiencias sensoriales.

La amplitud de tecnologías incorporadas a las estancias educativas hace que cada vez nos encontremos con entornos mejorados por estas (Technology Enhanced Learning Environment), en el sentido de que cualquier contexto que facilite la adquisición de conocimientos y habilidades puede ser considerado como un entorno de aprendizaje; y que cualquier entorno de aprendizaje que esté apoyado por las TIC puede considerarse como mejorado por la tecnología.

Las TIC han traído diferentes posibilidades para las instituciones educativas: ampliar los escenarios de formación, facilitar la aplicación de lo aprendido en diferentes contextos, romper la idea de que el aprendizaje se encuentra en los contenidos y más bien que se sitúan en la interacción y en las actividades que se producen alrededor de estos, así como el acceso a la información en diferentes lugares. Gusten o disgusten las TIC, hoy es imposible concebir escenarios de formación sin ellas.

Un problema que ha aparecido con las tecnologías es que no ha sido parejo su aumento de presencia con la mejora del rendimiento académico de los estudiantes, como han puesto de manifiesto diferentes investigaciones (Korte y Hüsing, 2006; Barrero-Osorio y Linden, 2009; European Commission, 2008). Esto se debe a una serie de aspectos, primero, hubo un tiempo en que - $-\mathrm{y}$ desgraciadamente para ciertos sectores continúa- se pensó que la simple incorporación de la tecnología en el aula implicaba su uso por docentes y discentes, y además que ello supondrá la transformación y mejora del proceso de enseñanzaaprendizaje. Ese solucionismo tecnológico, como diría Morozov (2015), no ha aportado grandes cosas; es más, muchas veces la incorporación de la TIC lo que ha supuesto es un verdadero refuerzo de pedagogías tradicionales y nada innovadoras, como ha ocurrido con la incorporación del PowerPoint, que más que servir como un elemento de guía y acompañamiento, se ha convertido en un organizador del proceso de enseñanza-aprendizaje, reforzando el papel del docente como transmisor de información y del discente como recolector de esta.

Hoy sabemos que la innovación con la tecnología ocurre cuando las incorporamos para que los alumnos alcancen un aprendizaje activo, abierto, flexible y colaborativo. Esto no depende exclusivamente ni de su presencia física ni de sus potencialidades tecnológicas, sino de cómo el docente las incorpora en la enseñanza, de las estrategias y metodologías que moviliza con estas y del tipo de interacción que pretendemos que establezcan los estudiantes con ellas, desde simplemente recordar la información a crearla a través de diferentes dispositivos tecnológicos.

Esto pasa por la forma en la cual el docente se apropia de la tecnología y

[...] se entiende la manera en que los docentes incorporan las TIC a sus actividades cotidianas de clase. La apropiación está en relación con el conocimiento que los docentes desarrollan sobre las TIC, el uso instrumental que hacen de ellas y las transformaciones que realizan para adaptarlas a sus prácticas educativas. (Valencia-Molina y SernaCollazos, 2016, p. 11).
Lo anterior sin lugar a duda es determinado por uno de los aspectos referidos posteriormente, como es el de la formación del profesorado.

El Gobierno escocés (Government Scottish, 2015) ha realizado recientemente una revisión de la literatura para explorar el uso que profesores, padres y alumnos hacen de las TIC en la enseñanza y el aprendizaje. En este pone de manifiesto que la utilización de la tecnología digital depende no solo de tener un número suficiente de acceso a los equipos, herramientas y recursos, sino también de la disponibilidad de contar con profesorado con formación, crear redes de conocimiento, establecer medidas de apoyo en los docentes para que comprendan los beneficios y aplicaciones de las TIC, y se les facilite su utilización, diseño y producción.

Este grado de no utilización depende de una serie de aspectos, algunos de los cuales se analizarán a continuación, pero antes se presentan las conclusiones a las que llegó un estudio realizado por la Fundación Telefónica (2016) efectuado en centros de toda España y en diferentes niveles educativos, considerados de buenas prácticas, donde se señala que estos centros comparten una serie de características comunes: 1) liderazgo y compromiso; 2) diseño de un plan de innovación; 3) aprendizaje basado en proyectos; 4) aulas cooperativas; 5) énfasis en las competencias; 6) encaje curricular en un marco innovador; 7) más allá del libro de texto; 8) rol del educador como guía y activador; 9) colaboración entre docentes y centros; 10) redefinición de los tiempos y espacios; 11) apertura al entorno; y 12) la tecnología como vía de integración y palanca de cambio. Como se observa, la simple presencia de las TIC no es suficiente para una enseñanza de calidad.

Para garantizar acciones exitosas con las TIC en las prácticas educativas debemos hacerlo desde diferentes perspectivas que superen su utilización únicamente para presentar información, como son: 1) alcanzar mejores o nuevos aprendizajes; 2) producir innovaciones educativas; 3 ) provocar cambios organizacionales; 4) extender los contenidos que pueden ser ubicados en la formación; 5) descontextualizar los escenarios de formación; y 6) facilitar los procesos de comunicación e interacción entre los participantes en el acto formativo. Tampoco se puede olvidar que ciertas tecnologías, como la realidad aumentada (Cabero y García, 2016), enriquecerán la información que ofrece la realidad.

Lo comentado lleva a señalar que uno de los aspectos importantes para la incorporación de las TIC no es plantear su utilización para mejorar las cosas que hacemos usualmente, sino fundamentalmente para hacer cosas diferentes. Ello requiere, por una parte, la formación y perfeccionamiento del docente, crear un currículo que se adecúe a la cultura digital y generar cambios en las prácticas pedagógicas y en las políticas de gestión. Lo que transforma la educación no es la incorporación de las TIC en los procesos de la enseñanza, sino los usos que específicamente se hacen de estas.

Mi punto de vista es que el foco debe estar menos puesto en las tecnologías y más en las nuevas prácticas culturales y mentalidades que han madurado alrededor de las nuevas herramientas y plataformas tecnológicas. En definitiva, ir hacia la búsqueda de aprender más, mejor y distinto. Esto lleva a mirar las TIC desde perspectivas diferentes a como tradicionalmente las hemos sopesados en el terreno educativo, de forma que de su concepción tradicional como TIC (Tecnologías de la Información y Comunicación), pasemos a observarlas como TAC (Tecnologías para el Aprendizaje y el Conocimiento) y como TEP (Tecnologías para el Empoderamiento y la Participación) (Cabero, 2014).

Movilizarlas como TAC implica su utilización como instrumentos facilitadores del aprendizaje y la difusión del 
conocimiento. Por lo tanto, habría que percibirlas más que como herramientas de comunicación, como materiales para la realización de actividades para el aprendizaje y el análisis de la realidad circundante por el estudiante. Se trata de dirigir su utilización hacia usos más formativos, tanto para el docente como el discente, con el objetivo de aprender más significativamente. Desde esta visión, su significación para la educación vendrá de las estrategias y metodologías que se aplicarán sobre estas para alcanzar los objetivos previstos y crear nuevas escenografías de comunicación para el aprendizaje; desde esta perspectiva, su incorporación debe potenciar la autonomía del estudiante. Al aplicarla como TAC se trataría de que el docente las movilice no para realizar lo mismo que hace sin estas —es decir, reproducir modelos tradicionales de enseñanza-, sino aplicarlas para crear innovaciones educativas y buscar en su implementación nuevos usos educativos, para que los alumnos las utilicen como instrumentos de formación y conocimiento, y no simplemente como herramientas tecnológicas e instrumentales.

Como TEP, se trataría de percibirlas no como meros recursos educativos, sino también como instrumentos para la participación y la colaboración de docentes y discentes. Es asumir que el aprendizaje tiene una fuerte dimensión social, porque la formación implica aprender en comunidad y ser capaz de interaccionar y colaborar para construir el conocimiento, donde la tecnología desempeña un papel de mediadora en la construcción del conocimiento y la interacción social.

Lo comentado implica percibir las TIC aplicadas en la enseñanza, comunicación, conocimiento, empoderamiento y participación; por lo tanto, su foco debe estar menos puesto en las tecnologías y más en las nuevas prácticas culturales y mentalidades que han madurado alrededor de las nuevas herramientas, plataformas tecnológicas, web 2.0 y los social media. Más que pensar en tecnologías futuras, lo que debemos es invertir el esfuerzo en inventar modelos de enseñanza para sacarles el máximo partido a las tecnologías que tenemos actualmente; además, las tecnologías ya no son solo de distribución de la información, sino más bien crean un protocolo de prácticas sociales y potencian nuevas formas de aprendizaje más colaborativas e interactivas.

La significación que las TIC están teniendo en nuestro entramado social hace que debamos repensar la escuela, de manera que potenciemos la valoración de lo imaginativo, se asigne mayor prioridad a la exploración y se otorgue más atención a lo distintivo sobre lo estandarizado, a lo metafórico sobre lo lineal, que le dé más prioridad a la interpretación que a la medición y finalmente encuentre más significativo la calidad del viaje que la velocidad con la que se ha llegado.

Ahora bien, la realización de estas prácticas académicas y culturales con las TIC implica, como se señaló en otro trabajo (Cabero, 2015, pp. 23-24), tener en cuenta una serie de principios e ideas, como por ejemplo:

- Cualquier tipo de TIC es simplemente un recurso didáctico que deberá ser movilizado cuando el alcance de los objetivos, contenidos, características de los estudiantes o proceso comunicativo lo justifique.

- $\quad$ El aprendizaje se encuentra no en función del medio, sino fundamentalmente sobre la base de las estrategias y técnicas didácticas que se movilicen.

- El profesor es el elemento más significativo a la hora de incorporarlas.

- Las TIC no solo transmiten información, sino que también transforman nuestra estructura cognitiva, de ahí que el mejor enfoque de enseñanza con estas sea multimedia.
- Antes de pensar en términos de qué medio, debemos pensar para quién, cómo se va a utilizar y qué se pretende con él.

- El contexto donde se incorpora una TIC condiciona su utilización.

- $\quad$ No existe el supermedio, todo medio es significativo para el proceso de enseñanza-aprendizaje.

- No usarlas exclusivamente para transmitir información, sino dar la oportunidad a los alumnos para con estas analizar el entorno circundante, y crear y elaborar contenidos.

Esto exigirá la aplicación de nuevas metodologías como: storytelling, gamificación, narrativa transmedia, cultura maker, aprendizaje colaborativo, flipped classroom, comunidades de aprendizaje o design thinking.

Lo anterior lleva a que el docente desempeñe roles diferentes a los movilizados en la escuela de la sociedad posindustrial, donde básicamente era transmisor de información y evaluador de los aprendizajes. En una sociedad donde los alumnos accederán a la información a través de diferentes fuentes, los roles a desempeñar serán diferentes a los ya citados, y concretamente se concentrarán en los siguientes:

- Consultores de información, facilitadores del aprendizaje.

- Diseñadores de situaciones mediadas de aprendizaje.

- Proveedor de contextos y escenografías formativas.

- Moderadores, tutores virtuales, orientadores y guías.

- Evaluador, seleccionador de tecnologías y curador de contenidos.

\section{Reflexiones finales}

Los obstáculos que podemos encontrar para la incorporación son de diverso calado, desde la formación del profesorado y el imaginario que tenemos sobre las tecnologías, hasta las estructuras organizativas donde se insertan. Sosa, Salinas y De Benito (2018) indican que nos encontramos con diferentes factores que pueden dificultar la incorporación de las tecnologías emergentes a la enseñanza. Las dificultades se señalan en diferentes tipos de niveles: microsistema (el profesor), mesosistema (condiciones de infraestructura y apoyo formal e informal a los profesores), exosistema (factores vinculados a la opinión de terceros, la experiencia y la satisfacción de otras personas) y macrosistema (políticas ministeriales).

Al mismo tiempo, uno de los errores más significativos es creer que las soluciones se refieren exclusivamente a la penetración e incorporación de TIC, cuando debe ser una combinación de medidas tecnológicas, políticas y pedagógicas, como en el ejemplo el informe elaborado por la Fundación Telefónica (2016) referido a la preparación de las escuelas para la sociedad digital.

En lo que se refiere al imaginario creado sobre las TIC, tanto desde la perspectiva de los apocalípticos como desde los integrados, de acuerdo con la denominación acuñada hace ya tiempo por Eco (1968), se ha mostrado una fuerte resistencia a los cambios que podrían traer la incorporación de las TIC en la enseñanza, aludiendo a su fuerte tecnificación y abandono del humanismo que requiere dicha actividad, o hasta quienes creen que la incorporación de las tecnologías per se mejorará la educación y que toda nueva tecnología es mejor que su predecesora. Tanto de una como de otra posición, se olvida que en las aulas siempre 
ha existido tecnología, ya que hasta el propio diseño de la acción educativa es en sí misma una acción tecnológica, pues la tecnología no funciona aisladamente del resto de variables del currículo, sino en estrecha relación con estas. Esta última es lo que determina su concreción, puesta en acción y los resultados de aprendizaje.

Como han puesto de manifiesto diferentes investigaciones, las actitudes que tenemos hacia las TIC condicionan las percepciones y usos que hagamos de estas y su grado de adopción, tanto por parte de los docentes como por los discentes (Vera et ál., 2014; Gallego et ál., 2016). Por otra parte, la formación que los docentes poseen respecto a las TIC (Almerich et ál., 2011, Suárez et ál., 2013; Roblizo y Cózar, 2015; Valdivieso y Gonzáles, 2016) se puede considerar de tipo medio, y menor en lo que serían las tecnologías más novedosas. Sin embargo, hay que resaltar dos aspectos: que su capacitación suele ser mayor en el terreno tecnológico que en el uso pedagógico; y que muestran dominios tecnológicos elevados en el uso que hacen de las tecnologías en su espacio doméstico y de investigación, y menos competentes cuando se refieren a prácticas educativas en la enseñanza (Prendes y Gutiérrez, 2013; Cabero y Marín, 2014; Rangel, 2015; Fernández,
Fernández y Cebreiro, 2016). Ahora bien, lo importante no es solo establecer planes de formación inicial y de perfeccionamiento del profesorado respecto a las TIC, sino desde dónde se deben efectuar. En ese sentido, una buena opción es la propuesta realizada por Mishra y Koehler (2006) conocida como modelo TPACK (Technological PedAgogical Content Knowledge), el cual indica que la capacitación de los docentes en TIC debe hacerse en tres tipos de conocimientos que deben estar interaccionados: conocimiento tecnológico, de contenido y pedagógico.

Por otro lado, el proceso de adopción por parte de los docentes de las TIC pasa por diferentes fases. Desde el proyecto ACOT (Apple Classrooms of Tomorrow), cuando durante años la empresa Apple dotó de infraestructura y ayuda tecnológica a diferentes centros de distintos estados norteamericanos (Fisher, 1988; Duyer, 1994; Salas-Castro y Martínez, 2014), con el ánimo de conocer el proceso que los docentes seguían para adoptarlas. Las conclusiones indican que la adopción de una tecnología pasa por distintas fases (figura 1), donde por lo general los docentes tardan de tres a cinco años en llegar a la fase de innovación.

Figura 1. Fases de adopción de las TIC por los docentes de acuerdo con el proyecto ACOT

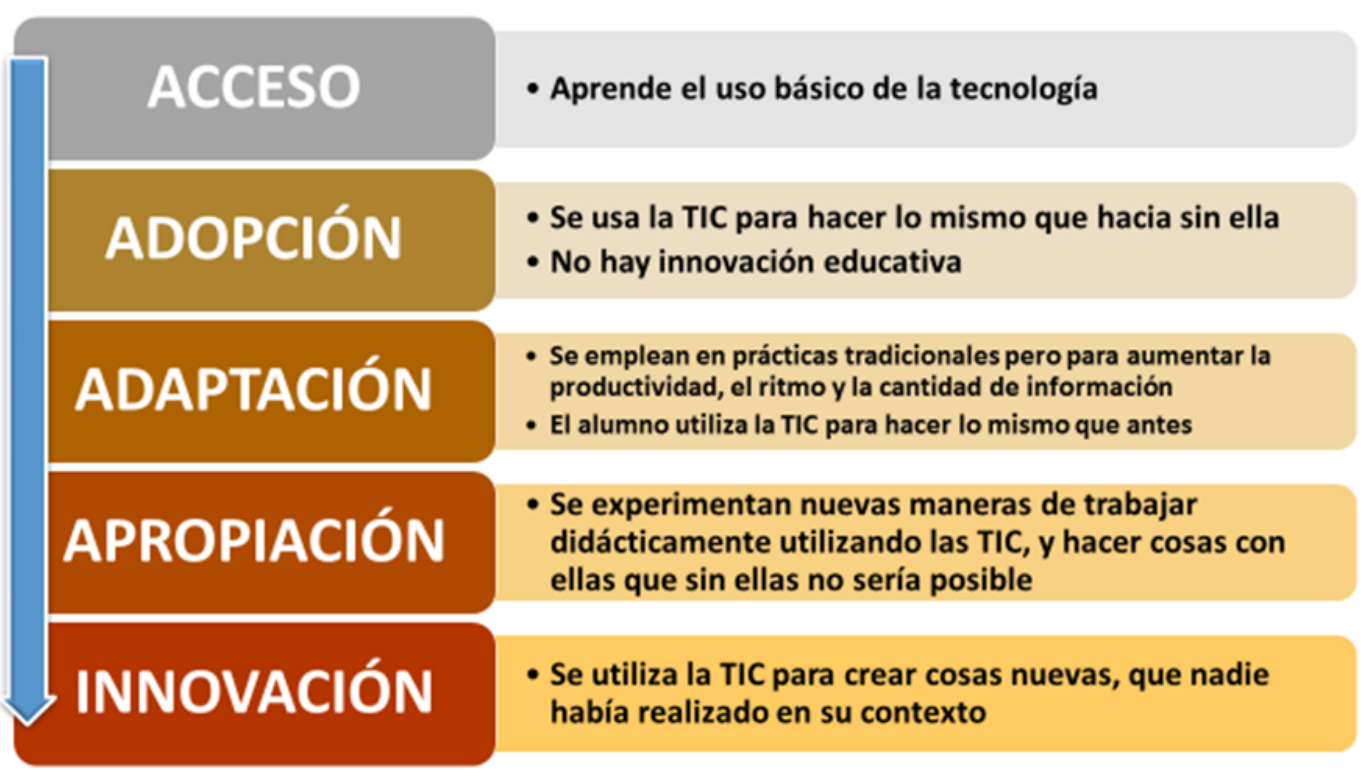

Fuente: elaboración propia.

En un trabajo desarrollado sobre formación del profesorado en TIC, indicamos que tal aprendizaje debe pasar por tres etapas: iniciación-instrumentación, incorporación-sustitución y revisióntransformación. En la primera, el profesor en activo o en formación toma contacto con las tecnologías y su aprendizaje instrumental. Por su parte, la segunda comienza cuando hay incorporación en su práctica educativa, inicialmente para sustituir determinadas acciones y con las tecnologías hacerlas más eficaces, eficientes y atractivas; y con la reflexión respecto al comportamiento que las TIC adquieren en los contextos reales de formación que llevan a una visión crítica —ni apocalíptica ni integrada - con respecto a las posibilidades de las TIC en los procesos formativos. Finalmente, en la tercera, que implica la transformación de la práctica educativa mediante la utilización de las TIC, se asume que no es solo un componente que puede movilizarse por el docente, sino que deben crearse prácticas que favorezcan a que el estudiante se convierta en proconsumidor de mensajes tecnológicos. Es el momento de buscar nuevas maneras de trabajar con las TIC en función de los diferentes contenidos disciplinares y de proponer e investigar nuevas formas de uso.

Cada una de estas etapas tendrá una tendencia hacia la formación en diferentes dimensiones: diseño, uso educativo, gestión-administración, investigación y ética, que deberán traducirse en estándares específicos y que serán más complejos cuando el docente pase por las diferentes etapas de apropiación técnica y conceptual de la tecnología (figura 2). 
Figura 2. Fases y dimensiones en la formación del docente en TIC

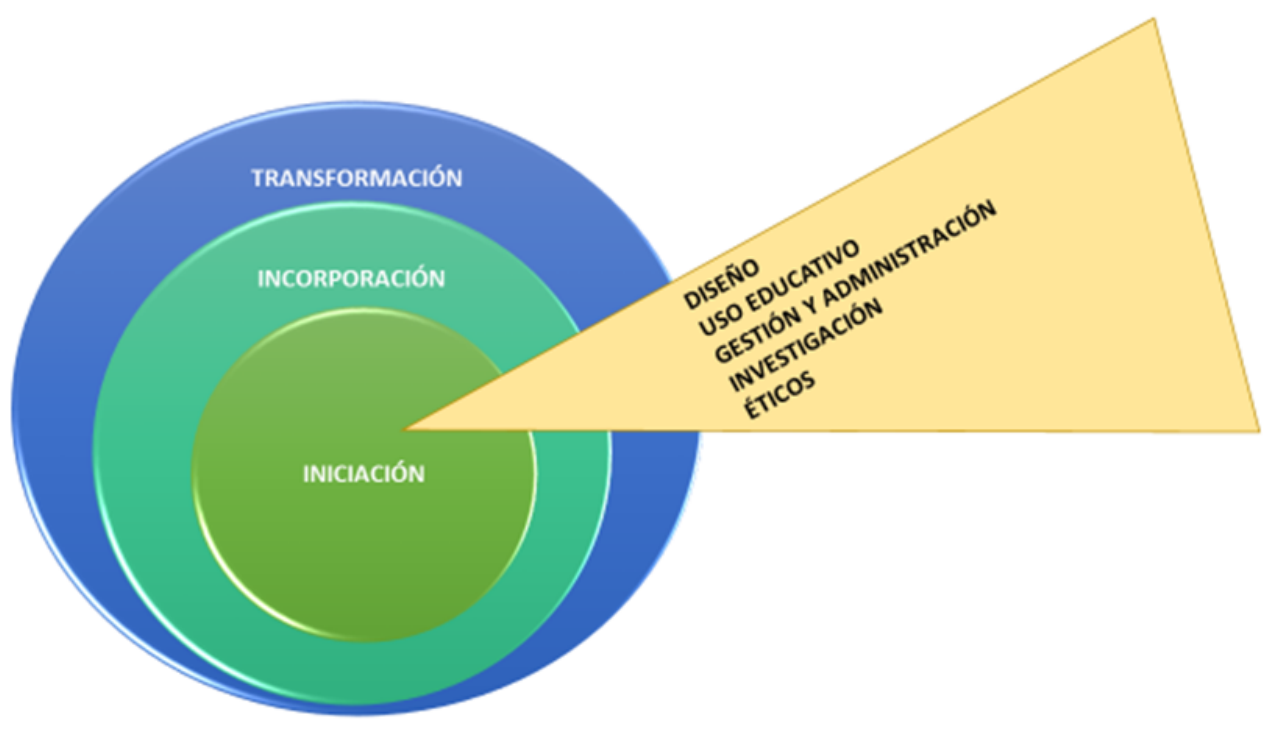

Fuente: elaboración propia.

Por otra parte, tenemos un nuevo tipo de alumno, y no se refiere a la clasificación de nativos digitales que ha llevado a muchas personas a creer que el simple hecho cronológico de la fecha de nacimiento los hace altamente competentes para el manejo de las TIC. Como han puesto de manifiesto diferentes investigaciones (Marín, Vásquez, Llorente y Cabero, 2012; Cabero, 2009; Cabra y Marciales, 2009; Romero y Minelli, 2011), los alumnos no son tan competentes tecnológicamente como se ha apuntado desde esa perspectiva y son más expertos rutinarios que estudiantes digitales, lo lleva a diferentes autores (Casati, 2015) a plantear que debemos ya olvidarnos de dicha denominación, por confusa e incierta y porque no todos los jóvenes tienen los mismos perfiles de dominio de las tecnologías, menos aun cuando nos movemos en el terreno de la formación. La Fundación Telefónica (2013), en el análisis de las 20 claves que proponen para el futuro de la educación, la séptima señala que hay que romper con el mito de que los nativos digitales dominan las TIC para uso y provecho en el siglo XXI.

Por eso, algunos autores apuntan que más que hablar de nativos e inmigrantes digitales deberíamos utilizar los términos visitantes y residentes: los primeros usan la red para buscar información o herramientas, recopilan información, les cuesta identificarse en la red y prefieren por lo tanto el anonimato, no creen en las redes por poco útiles, no desean perder el tiempo navegando y usan la red, pero no se identifican como miembros de esta. Por su parte, los segundos habitan el espacio en red donde encuentran relaciones de intereses y amistades, viven parte de su vida en línea, les cuesta distinguir entre lo real y lo virtual, pertenecen a varias comunidades virtuales y no se rehúsan a compartir su identidad digital.

$\mathrm{Al}$ asumir lo anterior, también debemos reconocer que invierten más tiempo en las tecnologías que las personas adultas (Ryberg et ál., 2011; Ruiz, 2016), que se sienten cómodos con estas y que prefieren lo audiovisual y telemático frente a lo impreso (Lai y Hong, 2015); pero de ahí a indicar que poseen altas competencias para movilizarlas desde un punto educativo e investigador hay un gran salto.

De todas maneras, esta amplia exposición a las tecnologías tiene consecuencias respecto a su estructuración cognitiva, como han llamado la atención diferentes autores (Carr, 2011 y Carr 2014; y Watson, 2011). De hecho, García Carrasco y Juanes (2013) afirman:

La tecnología que usamos cotidianamente es capaz de modificar nuestro cerebro mucho más de lo que creemos. Los hallazgos científicos de las últimas décadas han demostrado que el cerebro es un órgano plástico, constituido por neuronas capaces de regenerarse y de sufrir una remodelación permanente en respuesta a las experiencias que vivimos. (p. 48)

En un contexto tecnológico de sobreestimulación como el actual, los alumnos empiezan a presentar características cognitivas diferentes a los estudiantes de otro momento histórico, como, por ejemplo: son multitarea y tienden a pasar de forma rápida y urgente de una tarea a otra, necesitan la inmediatez en las acciones y resultados. Todo esto repercute en la pérdida de capacidad de atención y concentración, y en la dificultad para la movilización de un pensamiento profundo (Carr, 2011; 2014; y Watson, 2011). De ahí que los docentes manifiesten la dificultad para mantener el control de la clase, capturar la atención de sus estudiantes y hacer que se concentren en la realización de las tareas.

Otra de las dificultades es la falta de apoyo institucional con la que cuentan los profesores para la incorporación de las TIC, ya que desgraciadamente esta integración de las TIC tiende a recaer sobre los esfuerzos de los docentes. Cuando se les pregunta a los docentes sobre los problemas que suelen encontrarse para incorporar las TIC, hay un fuerte acuerdo en señalar la necesidad de que existan centros de apoyo para facilitar su producción y diseño. 
Nos enfrentamos a grandes retos que condicionarán las decisiones que adoptemos respecto a las tecnologías: formar a los estudiantes para trabajos no creados, para el uso de tecnologías que aún no han sido instauradas, formar para resolver problemas sociales económicos que aún no han surgido y para superar la brecha digital entre la sociedad/cultura e institución educativa/aula.

Para una incorporación positiva de las TIC en los contextos de formación se debe potenciar fuertemente su investigación y adoptar una posición crítica en los contextos formativos, ya que es más difícil traspasar ideas que tecnologías. Los cambios tecnológicos son rápidos y los educativos lentos y pausados. La historia de la incorporación de las TIC está llena de experiencias de tecnologías que serían el futuro, pero ni siquiera llegaron al presente.

\section{Referencias}

Almerich, G., Suárez-Rodríguez, J., Belloch, C. y Bo, R. M. (2011). Las necesidades formativas del profesorado en TIC: perfiles formativos y elementos de complejidad. Relieve. 17 (2), https: //doi.org/10.7203/relieve.17.2.4006

APS Group Scotland. (2015). Literature Review on the Impact of Digital Technology on Learning and Teaching. The Scottish Government.

Barrero-Osorio, F. y Linden, L. (2009). The use and misuse of computers in education: evidence from a randomized experiment in Colombia. The World Bank, Policy Research Working Paper Series.

Bauman, Z. (2007). Los retos de la educación en la modernidad líquida. Gedisa.

Brynjolfsson, E. y McAfee, A. (2014). The Second Machine Age. Work, Progress, and Prosperity in a Time of Brilliant Technologies. W. W. Norton \& Company.

Cabero, J. (2009). Capacitación digital de los alumnos de la Unidad Académica de Agronomía y Ciencias (Ciudad Victoria) de la Universidad Autónoma de Tamaulipas. Grupo de Investigación Didáctica. https://dialnet.unirioja.es/servlet/libro ?codigo $=705117$

Cabero, J. (2014). Nuevas miradas sobre las TIC aplicadas en la educación. Andalucía Educativa. 81 . http://www.juntadeandal ucia.es/educacion/webportal/web/revista-andalucia-educativa/ en-portada/-/noticia/detalle/nuevas-miradas-sobre-las-tic-aplic adas-en-la-educacion-julio-cabero-almenara-1

Cabero, J. (2015). Aplicación de las nuevas tecnologías al ámbito socioeducativo . IC editorial.

Cabero, J. (2016). Tendencias educativas para el siglo XXI . Ediciones CEF.

Cabero, J. y Barroso, J. (2013). La escuela en la sociedad de la información. La escuela 2.0. En J. Barroso y J. Cabero (coords.), Nuevos escenarios digitales (pp. 21-36). Pirámide.

Cabero, J. y García, F. (coords.) (2016). Realidad aumentada . Síntesis.

Cabero, J. y Llorente, M. C. (2010). Comunidades virtuales para el aprendizaje. Edutec . Revista Electrónica de Tecnología Educativa. 34 . http://edutec.rediris.es/Revelec2/revelec34/com unidades_vir- tuales_aprendizaje.html

Cabero, J. y Llorente, M. C. (2015). Tecnologías de la Información y la Comunicación (TIC): escenarios formativos y teorías del aprendizaje. Revista Lasallista de Investigación. 12 (2), 186-193.
Cabero, J. y Marín, V. (2014). Miradas sobre la formación del profesorado en tecnologías de información y comunicación (TIC).Enl@ce : Revista Venezolana de Información de,Tecnología y Conocimiento. 11 (2), 11-24.

Cabra, F. y Marciales, G. (2009). Nativos digitales: ¿ocultamiento de factores generadores de fracaso escolar? Revista Iberoamericana de Educación. 50 . http://www.rieoei.org/rie5 0a06.htm

Carr, N. (2011). ¿Qué está haciendo internet con nuestras mentes? Superficiales. Taurus.

Carr, N. (2014). Atrapados. Cómo las máquinas se apoderan de nuestras vidas. Taurus.

Casati, R. (2015). Elogio del papel. Contra el colonialismo digital . Ariel.

Castaño, C. y Cabero, J. (coords.) (2013). Enseñar y aprender en entornos de m.learning. Síntesis.

Conner, M. L. (2013). Introduction to Informal Learning . http:// marciaconner.com/resources/informal-learning/

Cross, J., Hart, J., Husband, J., Jarche, H., Jennings, C. y Quinn, C. (2010). Working Smarter in Terra Nova Circa 2015. eLearn, 2010, 8. https://doi.org/10.1145/1858579.1865480

Durall, E., Gros, B., Maina, M., Johnson, L. y Adams, S. (2012). Perspectivas tecnológicas: educación superior en Iberoamérica 2012-2017. The New Media Consortium. http://openaccess.uoc.edu/webapps/o2/bitstream/1 0609/17021/6/horizon_iberoamerica_2012_ESP.pdf

Duyer, D. (1994). Apple Classrooms of Tomorrow: What We've Learned. Educational Leadership. 51 (5), 4-10.

Eco, U. (1968). Apocalípticos e integrados . Lumen.

European Commission. (2008). The use of ICT to support innovation and lifelong learning for all . A report on progress . http://ec.europa.eu/education/lifelong-learning-programme/do c/sec2629.pdf

Fernández, C., Fernández, M. y Cebreiro, B. (2016). Desarrollo de un cuestionario de competencias en TIC para profesores de distintos niveles educativos. Píxel.Bit. Revista de Medios y Educación. 48, 135-148. https://www.redalyc.org/pdf/368/368 43409010.pdf

Fisher, Ch. (1988). The influence of High Computer Access on Schoolwork and Student Empowerment: An Exploratory Study of the Nashville ACOT Site . Apple Computer, Inc.

Floridi, L. (2017). The Fourth Revolution: How the Infosphere Is Reshaping Human Reality . Oxford University Press.

Fundación Telefónica (2013). Telefónica Global Millennial Survey: Global Results . Fundación Telefónica. http://survey.telefonica.com/globalreports/assets/T elefonica\%20-\%20Global\%20Millennial\%20Survey.pdf

Fundación Telefónica (2016). Prepara tu escuela para la sociedad digital . Fundación Telefónica.

Gallego, O., Cabero, J. y Sampedro, B. (2016). Valoraciones de la 'aceptación de la tecnología de formación virtual' por profesores universitarios asistentes a un curso de formación virtual. EDUTEC. Revista Electrónica de Tecnología Educativa, 56, a335-a335. https://doi.org/10.21556/edutec.201 6.56 .745

García Carrasco, J. y Juanes, J. A. (2013). El cerebro y las TIC. Teoría de la Educación. Educación y Cultura en la Sociedad de la Información. 14 (2), 42-84.

García, I., Peña-López, I., Johnson, L., Smith, R., Levine, A. y Haywood, K. (2010). Informe Horizon: 
Edición Iberoamericana 2010. The New Media Consortium. http://openaccess.uoc.edu/webapps/o2/bitstream/1 0609/2661/6/NMC_HorizonReport_IB_2010_def.pdf

García, M. (2017). El fin del mundo tal y como lo conocemos . Planeta.

Gardner, H. (1998). Inteligencias múltiples . Paidós.

González, F. (2017). De la era de la perplejidad a la era de las oportunidades: finanzas para el crecimiento. En BBVA. La era de la perplejidad. Repensar el mundo que conocíamos (pp. 8-39). Taurus.

Government Scottish. (2015). Literature Review on the Impact of Digital Technology on Learning and Teaching . https://www.gov.scot/publications/literature-review-impact-di gital-technology-learning-teaching/

Jarvis, J. (2015). El fin de los medios de comunicación de masas . Gestión 2000

Johnson, L., Smith, R., Levine, A. y Stone, S. (2010). The 2010 Horizon Report: Edición en español (Xavier Canals, Eva Durall, traducción). The New Media Consortium. https://www. lacosox.org/sites/default/files/2010-Horizon-Report-es_0.pdf

Johnson, L., Adams Becker, S., Cummins, M., Estrada, V., Freeman, A., y Hall, C. (2016). NMC Informe Horizon 2016 Edición Superior de Educación. The New Media Consortium. http://www.aprendevirtual.org/centro-documentac ion-pdf/2016-nmc-horizon-report-HE-ES.pdf

Korte, W. B. y Hüsing, T. (eds.) (2006). Benchmarking access and use of ICT in European schools 2006: Final report from head teacher. And classroom teacher surveys in 27 European countries . Empirica Gesellschaft für Kommunikations-und Technologieforschung.

Lai, K-W. y Hong, K-S. (2015). Technology use and learning characteristics of students in higher education: Do generational differences exist? British Journal of Educational Technology. 46 (4), 725-738. https://doi.org/10.1111/bjet.12161

Ljubojevic, M., Vaskovic, V., Stankovic, S. y Vaskovic, J. (2015). El uso de video complementario en la enseñanza multimedia como herramienta didáctica para incrementar la eficiencia del aprendizaje y la calidad de experiencia. Revista Mexicana de Bachillerato a Distancia, .(13), 20. http://dx.doi.org/10.22201/ cuaed.20074751e.2015.13.64999

Loveless, A. y Williamson, B. (2017). Nuevas identidades de aprendizaje en la era digital . Narcea.

Marín, V., Vásquez, A., Llorente, M. y Cabero, J. (2012). La alfabetización digital del docente universitario en el espacio europeo de Educación Superior. EDUTEC. 39, 1-10. https://id us.us.es/bitstream/handle/11441/24662/file_1.pdf?sequence $=1$

Mas, X. (2017). El tejido de Weiser. Claves, evolución y tendencias de la educación digital . OuterEDU.

Mishra, P. y Koehler, M. (2006). Technological Pedagogical Content Knowledge: A Framework for Teacher Knowledge. Teachers College Record. 108, 1017-1054.

Morozov, E. (2015). La locura del solucionismo tecnológico . Kartz Editores.

Organizacio\#n de Estados Iberoamericanos (OEI). (2010). 2021. Metas educativas. La educación que queremos para la generación de los bicentenarios. OEI.

Organización para la Cooperación y el Desarrollo Económicos [OCDE], y Junta de Extremadura. (2008). El conocimiento libre y los recursos educativos abiertos. Junta de Extremadura. htt ps://www.oecd.org/spain/42281358.pdf
Pérez Gómez, A. (2012). Educarse en la era digital. La escuela educativa. Morata.

Prendes, M. P. y Gutiérrez, I. (2013). Competencias tecnológicas del profesorado en las universidades españolas. Revista de Educación. 361 , 196-222.

Prensky, M. (2011). Enseñar a nativos digitales . SM.

Prieto, A. (2017). Flipped learning: aplicar el modelo del aprendizaje inverso . Narcea.

Rangel, A. (2015). Competencias docentes digitales: propuestas de un perfil. Píxel-Bit. Revista de Medios y Educación. 46 , 235-248.

Rifkin, J. (2014). La sociedad de coste marginal cero . Paidós.

Roblizo, M. y Cózar, R. (2015). Usos y competencias en TIC en los futuros maestros de educación infantil y primara: hacia una alfabetización tecnológica real para docentes. Píxel-Bit. Revista de Medios y Educación. 47, 23-39.

Rojas, L. (2011). Eres tu memoria. Conócete a ti mismo . Espasa.

Romero, M. y Minelli, J. (2011). La generación net se tambalea. Percepción del dominio de las TIC de estudiantes de magisterio. Revista Teoría de la Educación: Educación y Cultura en la Sociedad de la Información. 12 (3), 265-283.

Rué, J. (2015). Entornos de aprendizaje digitales y calidad de la educación superior. UOC.

Ruiz, C. (2016). Perfil del uso del teléfono móvil e internet en una muestra de universitarios españoles: ¿usan o abusan? Bordón. 68 (3), 131-145.

Ryberg, T., Dirckinck-Holmfeld, L. y Jones, C. (2010). Catering to the Needs of the "Digital Natives" or Educating the "Net Generation"? Web 2.0-Based E-Learning: Applying Social Informatics for Tertiary Teaching, 301-318. https://doi.org/10 .4018/978-1-60566-294-7.ch016

Salas-Castro, R. y Martínez, J. (2014). Aulas Apple del mañana: resultados empíricos de educación básica (Modelo ACOT). En Esquivel-Gámez (coords.), Los modelos tecno.educativos: revolucionando el aprendizaje del siglo XXI (pp. 17-32). DSAE; Universidad Veracruzana.

Salmi, H. (2012). Evidence of bridging the gap between formal education and informal learning through teacher education. Reflecting Education,.(2), 45-61.

Sosa, E., Salinas, J., y de Benito, B. (2017). Emerging Technologies (ETs) in Education: A Systematic Review of the Literature Published between 2006 and 2016. International Journal of Emerging Technologies in Learning (IJET), 12(5), 128-149. doi:10.3991/ijet.v12i05.6939

Sosa, E., Salinas, J. y De Benito, B. (2018). Factors that facilitate or limit the incorporation of emerging technologies in the classroom. International Online Journal of Education and Teaching (IOJET), 5(1), 38-59.

Suárez, J., Almerich, G., Gallardo B. y Aliaga, F. (2013). Las competencias del profesorado en TIC: estructura básica. Educación XX1, 16(1), Article 1. https://doi.org/10.5944/educx $\mathrm{x} 1.16 .1 .716$

Tecnológico de Monterrey. (2015). Reporte EduTrends. Radar de Innovación Educativa 2015 . Tecnológico de Monterrey.

Tünnerman, C. (2009). La universidad del siglo XXI . Universidad Juárez del Estado de Durango.

Unesco. (2013). Directrices para políticas de desarrollo y promoción del acceso abierto. Unesco.

Unesco. (2015). Replantear la educación ¿Hacia un bien común mundial? Unesco. 
Unión Internacional de Telecomunicaciones. (2003). Informe sobre el desarrollo mundial de las telecomunicaciones. UIT. https://www.itu.int/ITU-D/ict/publications/wtdr_03/mater ial/WTDR03Sum_s.pdf

Valdivieso, T. y González, M. A. (2016). Competencia digital docente: ¿dónde estamos? perfil del docente de educación primaria y secundaria. El caso de Ecuador. Píxel-Bit. Revista de Medios y Educación. 49, 57-73.

Valencia, R. (2017). Reformateando la universidad, U.gob. Tecnología en Gobierno . https://u-gob.com/reformateando-launiversidad/

Valencia, R. (2018). Empleo del futuro. U.gob. Tecnología en Gobierno . https://u-gob.com/empleos-del-futuro/

Valencia-Molina, T y Serna-Collazos, A. (2016). Competencias $y$ estándares TIC desde la dimensión pedagógica: una perspectiva desde los niveles de apropiación de las TIC en la práctica educativa docente . Pontificia Universidad Javeriana.

Vázquez-Cano, E. y Sevillano, M. L. (eds.) (2015). Dispositivos digitales móviles en educación. Narcea.

Vera, J., Moran, L. y García, E. (2014). Evaluación de competencias básicas en TIC en docentes de Educación Superior en México. Pixel-Bit Revista de Medios y Educación 1133 (8482), 143-155. https://doi.org/10.12795/pixelbit.2014.i 44.10

Watson, R. (2011). Mentes del futuro . Vicerversa.

West, D. (2016). Megachange. Economic Disruption, Political Upheaval, and Social Strife in the 21 st Century. The Brookings Institution.

\section{Bibliografía consultada}

Castells, M. (1998). La era de la información. Economía, sociedad y cultura, Fin del milenio. Alianza.

Echeverría, J. (199). Los señores del aire: Telépolis y el tercer entorno. Destino.

Hawkey, R. (2002). The lifelong learning game: season ticket or free transfer? Computers \& Education, 38, 5-20.

Joyanes, L. (1997). Cibersociedad. Los retos ante un nuevo mundo digital. McGraw-Hill.

The Scottish Government. (2016). Enhancing learning and teaching through the use of digital technology. The Scottish Government.

Touraine, A. (1969). La societé pos-indsutrielle. Denoël.

Notas

* Ponencia presentada en el II Coloquio Nacional y I Internacional de Estudiantes de Doctorado y Posdoctorado en Educación: la educación superior de cara al presente y futuro de América Latina. 